\title{
Some new aspects of main eigenvalues of graphs
}

\author{
Nair Abreu ${ }^{1}$ (D) - Domingos M. Cardoso ${ }^{2}$ (D) Francisca A. M. França ${ }^{3}$ (D) \\ Cybele T. M. Vinagre ${ }^{4}(\mathrm{D}$
}

Received: 6 August 2018 / Revised: 29 September 2019 / Accepted: 13 October 2019 /

Published online: 1 November 2019

(c) SBMAC - Sociedade Brasileira de Matemática Aplicada e Computacional 2019

\begin{abstract}
An eigenvalue of the adjacency matrix of a graph is said to be main if the all-1 vector is non-orthogonal to the associated eigenspace. This paper explores some new aspects of the study of main eigenvalues of graphs, investigating specifically cones over strongly regular graphs and graphs for which the least eigenvalue is non-main. In this case, we characterize paths and trees with diameter-3 satisfying the property. We may note that the importance of least eigenvalues of graphs for the equilibria of social and economic networks was recently uncovered in literature.
\end{abstract}

Keywords Main eigenvalue $\cdot$ Cone $\cdot$ Harmonic graph $\cdot$ Path $\cdot$ Double star

Mathematics Subject Classification 05C50 $\cdot 05 \mathrm{C} 05 \cdot 05 \mathrm{C} 38$

Communicated by Carlos Hoppen.

The research of Domingos M. Cardoso is partially supported by the Portuguese Foundation for Science and Technology ("FCT-Fundação para a Ciência e a Tecnologia "), through the CIDMA-Center for Research and Development in Mathematics and Applications, within project UID/MAT/ 04106/2019. This author also thanks the support of Project Universal CNPq 442241/2014 and Bolsa PQ 1A CNPq, 304177/2013-0.

Cybele T. M. Vinagre

cybele_vinagre@id.uff.br

Nair Abreu

nairabreunovoa@gmail.com

Domingos M. Cardoso

dcardoso@ua.pt

Francisca A. M. França

francisca_franca@id.uff.br

1 PEP/COPPE, Universidade Federal do Rio de Janeiro, Rio de Janeiro, Brazil

2 Department of Mathematics, Center for Research and Development in Mathematics and Applications, Universidade de Aveiro, 3810-193 Aveiro, Portugal

3 Instituto de Ciências Exatas, Universidade Federal Fluminense, Volta Redonda, Brazil

4 Instituto de Matemática e Estatística, Universidade Federal Fluminense, Niterói, Brazil 


\section{Introduction}

Cvetković (1970) introduced the concept of main eigenvalue of a graph, that is, an eigenvalue of its adjacency matrix for which the associated eigenspace is non-orthogonal to the vector whose entries are equal to 1 (otherwise, it is called non-main). As a consequence of PerronFrobenius' Theorem, it is well known that a graph $G$ on $n \geq 2$ vertices is connected if and only if its largest eigenvalue (index) is simple and the associated eigenvector has only positive entries, so it is main, see Cvetković et al. (1979, Ths. 0.3, 0.4). We also note that a graph is regular if and only if it has only one main eigenvalue, see Cvetković (1971). In Huang et al. (2015), a method to construct families of graphs with exactly $s>1$ main eigenvalues is given; however, the characterization of such graphs is a problem proposed by Cvetković (1978) that remains open.

In Sect. 2, some properties of the graphs with exactly two main eigenvalues are studied. The two main eigenvalues of a cone over a strongly regular graph are identified and conditions for such a cone to be multiplicative are given. Also, harmonic graphs are characterized through its main spectral properties without any restriction on its combinatorial structure.

Cvetković (1971) started the investigation on the relation between the main eigenvalues of a graph and the eigenvalues of its complement. This subject was also approached in Hagos (2002), Teranishi (2002), and Cardoso and Pinheiro (2009). Particularly in Cardoso and Pinheiro (2009), such relations were investigated seeking estimates for the maximal size of regular induced subgraphs in the context of convex quadratic programming. At the end of the paper, three questions are raised. The first wonders about the existence of a connected graph $G$ whose complement $\bar{G}$ has a main eigenvalue between its largest eigenvalue and $-1-\lambda_{\min }(G)$, where $\lambda_{\min }(G)$ denotes the least eigenvalue of $G$. The second raises the possibility of characterizing graphs $G$ whose spectrum of $\bar{G}$ contains $-1-\lambda_{\min }(G)$ as a main eigenvalue. The third question approaches the characterization of connected graphs for which the least eigenvalue is non-main.

In Sect. 3, the first question posed in Cardoso and Pinheiro (2009) is discussed. The largest and the second largest eigenvalues of the complement of a graph are also analyzed, and we conclude that $-1-\lambda_{\min }(G)$ belongs to the spectrum of $\bar{G}$ if and only if it coincides with its second largest eigenvalue. Moreover, we show that, among all connected bipartite graphs, the balanced complete bipartite graphs $K_{r, r}$ are those whose respective complements contain $-1-\lambda_{\min }(G)$ as a main eigenvalue.

In Sect. 4, we determine the main spectrum, i.e., the set of distinct main eigenvalues, of a path with $n$ vertices and conclude that the least eigenvalue of such graph is non-main if and only if $n$ is even. On the other hand, the main eigenvalues of arbitrary diameter- 3 trees ( double stars) are determined and it is shown that their main spectra have cardinality four when they are not balanced. Finally, we conclude that among such trees, only the balanced ones have the least eigenvalue non-main.

After completing the present work, its authors became aware of the recent article of Bramoullé et al. (2014), which announced itself to be the first to uncover the importance of the least eigenvalue of a graph to economic and social outcomes showing the way this parameter "captures how much the network amplifies agents' actions". In the same direction, an recent paper of Allouch (2017) investigates the welfare effect of income redistribution in the private provision of public goods on networks. Their analysis leads to the conclusion that, as far as the direction of welfare-improving transfers is concerned, networks where the least eigenvalue is a main eigenvalue may have an useful policy implication. The mentioned article Allouch (2017) cites our examples displayed in Sections 3 and 4, which can also be 
found in a preliminar version of the present paper (Abreu N, Cardoso D, França F, Vinagre C On main eigenvalues of certain graphs. arXiv:1605.03533v1 [math.CO]).

Throughout this paper, unless otherwise stated, $G$ denotes a simple graph of order $n$ with edge set $E(G)$ and vertex set $V(G)=\{1, \ldots, n\}$. By $N_{G}(i)$, we indicate the set of neighbors of the vertex $i$. As usually, $P_{n}$ and $K_{n}$ denote, respectively, the path and the complete graph of order $n$. The adjacency matrix of $G, \mathbf{A}=\left[a_{i j}\right]$, is the $n \times n$ matrix for which the entries are $a_{i j}=1$, if $i j \in E(G)$, and 0 otherwise. The eigenvalues of $\mathbf{A}$ are also called the eigenvalues of $G$. Unless otherwise stated, the eigenvalues of $G$ are considered in non-increasing order, that is, $\lambda_{\max }=\lambda_{1} \geq \lambda_{2} \geq \cdots \geq \lambda_{n}=\lambda_{\min }$. When necessary, we write $\mathbf{A}(G)$ instead of $\mathbf{A}$ and $\lambda_{i}(G)$ instead of $\lambda_{i}$, for $i \in\{1,2, \ldots, n\}$. The eigenspace associated with the eigenvalue $\lambda(G)$ is denoted by $\varepsilon_{G}(\lambda)$. We recall that a partition $\pi$ of the set $V(G)$ of the graph $G$ is equitable when, given two cells $V_{i}$ and $V_{j}$ of $\pi$, there is a constant $m_{i j}$, such that each vertex $v \in V_{j}$ has exactly $m_{i j}$ neighbors in $V_{j}$. The matrix $\mathbf{M}=\left[m_{i j}\right]$ is called the quotient matrix of $G$ with respect to $\pi$. It is known (Th. 3 of Cvetković 1978) that the main eigenvalues of $G$ are eigenvalues of $\mathbf{M}$. The graph $G$ is said to be equitable when its valency partition, that is, the partition of $V(G)$ according the vertex degrees, is equitable. The all one $n \times n$ matrix is denoted $\mathbf{J}$ and $\mathbf{j}$ denotes a column of the matrix $\mathbf{J}$.

For basic notions and notation from spectral graph theory not herein defined, the reader is referred to the book by Cvetković et al. (2010) or Brouwer and Haemers (2012).

\section{Graphs with exactly two main eigenvalues}

Graphs with just two main eigenvalues have been studied in several papers as the works by Lepović (2001), Hagos (2002), Teranishi (2002), Hou and Tian (2006), Nikiforov (2006), and Rowlinson (2007). Namely, in Rowlinson (2007), a complete survey on the main eigenvalues of a graph (up to 2007) is given and the graphs with exactly two main eigenvalues deserve particular attention. New results on these graphs appear in more recent publications as the works by Cardoso et al. (2010), Shi (2009), Hu et al. (2009), and Hayat et al. (2016, 2017).

Let us call the graphs with the same main eigenvalues co-main-spectral graphs. Infinite families of non-isomorphic co-main-spectral graphs with exactly two main eigenvalues appear in the paper by Cardoso et al. (2010). For instance, the biregular graphs $H_{k}^{q}$ obtained from a connected $k$-regular graph $H_{k}$ of order $p$ after attaching $q \geq 1$ pendent vertices to each vertex of $H_{k}$ (then the order of $H_{k}^{q}$ is $n=(q+1) p$ ) were considered. All of these graphs (independently of $p$ ) have exactly the two main eigenvalues $\lambda_{i}\left(H_{k}^{q}\right)=\frac{k \pm \sqrt{k^{2}+4 q}}{2}$, $i=1,2$. For $k=2, H_{2}$ is the cycle $C_{p}$, and for all $p \geq 3$

$$
\lambda_{1}\left(H_{2}^{q}\right)=1+\sqrt{1+q} \text { and } \lambda_{2}\left(H_{2}^{q}\right)=1-\sqrt{1+q}
$$

are their two main eigenvalues. Notice that for $p=3,4, \ldots$, we obtain an infinite sequence of co-main-spectral graphs of increasing order equal to $(q+1) p$ [see Cardoso et al. (2010, Fig. 2)].

The cone over $G$ is the join $K_{1} \nabla G$; that is, the graph obtained by adding a vertex to $G$ and connecting this vertex to all the vertices of $G$. When $G$ is regular and non-complete, the cone over $G$ is a biregular equitable graph. According to a result by Hayat et al. (2016, Th. 2.1(b)), it has two main eigenvalues. Furthermore, if $\mathscr{G}_{k, n}$ denotes the family of connected $k$-regular non-complete graphs of order $n$, we may conclude the following proposition. 
Proposition 1 If $H \in \mathscr{G}_{k, n}$, then the cone $K_{1} \nabla H$ has exactly the following two main eigenvalues:

$$
\rho, \theta=\frac{k \pm \sqrt{k^{2}+4 n}}{2} .
$$

Moreover, with the exception of the index, each eigenvalue of $H$ remains as an eigenvalue of the cone $K_{1} \nabla H$ with at least the same multiplicity.

Proof Let us assume that $\tau$ is a main eigenvalue of $G=K_{1} \nabla H$ with an associated eigenvector $\mathbf{v}=\left[\begin{array}{l}\beta \\ \mathbf{u}\end{array}\right]$ non-orthogonal to $\left[\begin{array}{l}1 \\ \mathbf{j}\end{array}\right]$, where $\beta$ is a constant to be determined. Then, it follows that:

$$
\mathbf{A}(G) \mathbf{v}=\left[\begin{array}{cc}
0 & \mathbf{j}^{\top} \\
\mathbf{j} & \mathbf{A}(H)
\end{array}\right]\left[\begin{array}{l}
\beta \\
\mathbf{u}
\end{array}\right]=\beta\left[\begin{array}{l}
0 \\
\mathbf{j}
\end{array}\right]+\left[\begin{array}{c}
\mathbf{j}^{\top} \mathbf{u} \\
\mathbf{A}(H) \mathbf{u}
\end{array}\right]=\tau\left[\begin{array}{l}
\beta \\
\mathbf{u}
\end{array}\right] .
$$

Therefore, $\beta \mathbf{j}+\mathbf{A}(H) \mathbf{u}=\tau \mathbf{u}$ and $\mathbf{j}^{\top} \mathbf{u}=\tau \beta$, and then, we have $\beta \neq 0$ and $\tau \neq 0$. Furthermore, from $\mathbf{j}^{\top}(\beta \mathbf{j}+\mathbf{A}(H) \mathbf{u})=\tau \mathbf{j}^{\top} \mathbf{u}$, it follows that $\beta n+k \tau \beta=\tau^{2} \beta$, and then, $\tau^{2}-k \tau-n=0$. Thus:

$$
\tau_{1}, \tau_{2}=\frac{k \pm \sqrt{k^{2}+4 n}}{2},
$$

(and $\beta_{i}=\frac{1}{\tau_{i}} \mathbf{j}^{\top} \mathbf{u}, i=1,2$ ). Taking into account that any eigenvalue $\alpha$ of $H$ with associated eigenvector $\mathbf{w}$ non-orthogonal to $\mathbf{j}$ is an eigenvalue of $\mathbf{A}(G)$ with eigenvector $\left[\begin{array}{l}0 \\ \mathbf{w}\end{array}\right]$, the last part follows.

Remark 1 As immediate consequence of Proposition 1, it follows that the cones over graphs in $\mathscr{G}_{k, n}$ are co-main-spectral with main eigenvalues given by (1).

It should be noted that if $H \in \mathscr{G}_{k, n}$ and $\theta=\frac{k-\sqrt{k^{2}+4 n}}{2}$ is an eigenvalue of $H$ (and thus, it is non-main), then $\theta$ becomes a main eigenvalue for the cone $K_{1} \nabla H$. Therefore, in this cone, the number of distinct eigenvalues remains the same as in the graph $H$. We can give examples of this situation for some strongly regular graphs $H$. We may recall that a graph of order $n$ is called strongly regular with parameters $(n, k, a, c)$ (more simply, a $(n, k, a, c)$-srg) when it is $k$-regular, each pair of vertices has $a$ or $c$ common neighbors depending whether they are adjacent or non-adjacent and the graph is not complete or edgeless. Such graphs have exactly three distinct eigenvalues, say $k>\mu>\lambda$, being that only $\lambda$ is negative. From the eigenvalues of $H$, their parameters may be obtained as follows (see Cvetković (1971, Th. 3.6.4)):

$$
\begin{aligned}
& n=\frac{(k-\mu)(k-\lambda)}{k+\mu \lambda}, \\
& a=k+\mu \lambda+\mu+\lambda, \\
& c=k+\mu \lambda .
\end{aligned}
$$

Thus, if $H$ is a strongly regular graph of order $n$ with eigenvalues $k>\mu>\lambda$, from (1), it follows that $K_{1} \nabla H$ remains having three distinct eigenvalues if and only if $-\lambda(k-\lambda)=n$ as it was stated by Muzychuk and Klin (1998, Prop. 6.1(b)). Theorem 1 (item 2), in the next subsection, gives an alternative necessary and sufficient condition in terms of the parameters of the strongly regular graph $H$. Now, we are ready to present some examples of graphs of $\mathscr{G}_{k, n}$ with $\theta$ as an eigenvalue. Let $H$ be one of the following graphs: the Petersen graph, the 
Shrikhande graph, the Gewirtz graph, or any one of the three Chang graphs. The first one is a $(10,3,0,1)$-srg that belongs to $\mathscr{G}_{3,10}$, the second graph is a $(16,6,2,2)$-srg belonging to $\mathscr{G}_{6,16}$, the third one is a $(56,10,0,2)$-srg in the class $\mathscr{G}_{10,56}$, and the three Chang graphs are $(28,12,6,4)$-srg belonging to $\mathscr{G}_{12,28}$.

\subsection{Cones over strongly regular graphs}

The characterization of graphs with three distinct eigenvalues is a challenging problem in Combinatorics that remains open, since the question posed by Haemers, during the 15th British Combinatorial Conference (Stirling, July 1995): are there any connected graphs apart from strongly regular and complete bipartite graphs which have just three distinct eigenvalues? Haemers' question was investigated by several researchers, but, among the published results, the non-regular connected graphs with just three distinct eigenvalues all of them not less than -2 were characterized in the paper by van Dam $(1998, T h .7)$ and those graphs with index less than 8 were characterized by Chung and Omidi (2009) [a particular family of cones had already been obtained by Mena and Bridges (1981), in 1981]. More recently, the characterization of connected biregular graphs with three distinct eigenvalues and second largest eigenvalue equal to 1 appeared in the paper by Cheng et al. (2016, Prop. 4.11) and in Cheng et al. (2018), the connected graphs with three distinct eigenvalues and second largest eigenvalue at most 1 were characterized. In Rowlinson (2016, 2017), some particular graphs were determined using the concept of main/non-main eigenvalue.

Now, it is worth recalling a remark that appears in Rowlinson (2016) as a consequence of results by van Dam (1998, Prop. 2 and 3), herein stated as the following lemma.

Lemma 1 (Rowlinson 2016) A non-integral connected graph $G$ with three distinct eigenvalues is either complete bipartite or its two smaller eigenvalues are algebraic conjugates. In the later case, $G$ has exactly 1 or 3 main eigenvalues.

Proposition 2 If $G$ is a connected non-complete bipartite graph with three distinct eigenvalues where two of them are main, then $G$ is a biregular integral graph.

Proof Let $G$ be a connected graph with three distinct eigenvalues two of which are main. Then, it is immediate that $G$ is biregular, see Rowlinson (2016, Lem. 2.2). If we assume that $G$ is non-integral, since it is non-complete bipartite, applying Lemma $1, G$ has exactly 1 or 3 main eigenvalues, which is a contradiction.

A strongly regular graph is called primitive if both the graph and its complement, which is also a strongly regular graph, are connected; otherwise, it is called imprimitive. A strongly regular graph with parameters $(n, k, a, c)$ is imprimitive if and only if $c=k$ or $c=0$. Thus, the eigenvalues of a primitive strongly $k$-regular graph satisfy $k>\mu>0$ and $\lambda<-1$. For more details, see Brouwer and Haemers (2012). Combining (1) and known facts about strongly regular graphs, we have the following result.

Theorem 1 Let $H$ be a primitive strongly regular graph with parameters $(n, k, a, c)$ and distinct eigenvalues $k>\mu>\lambda$. Then

1. if $H$ is non-integral, the cone $K_{1} \nabla H$ has more than three distinct eigenvalues;

2. $K_{1} \nabla H$ has three distinct eigenvalues $\rho>\mu>\lambda$ if and only if $\sqrt{k^{2}+4 n}-\sqrt{(a-c)^{2}+4(k-c)}=k-(a-c)$. 
Proof 1. Let us suppose that $K_{1} \nabla H$ has three eigenvalues. Since $H$ is connected and $k$ regular, the cone $K_{1} \nabla H$ has exactly two main eigenvalues, by Proposition 1. Furthermore, since $H$ is non-integral and $K_{1} \nabla H$ is not bipartite, according to Lemma 1, the two smaller eigenvalues of $K_{1} \nabla H$ are algebraic conjugates and, so, it has exactly one or three main eigenvalues, which is a contradiction.

2. It is well known that the eigenvalues of a $(n, k, a, c)$-srg like $H$ are $k$ and the roots of the polynomial $x^{2}-(a-c) x-(k-c)=0$. Therefore, it follows that the least eigenvalue of $H$ is given by the expression:

$$
\lambda=\frac{(a-c)-\sqrt{(a-c)^{2}+4(k-c)}}{2} .
$$

Taking into account Proposition 1 and the fact that $K_{1} \nabla H$ has three distinct eigenvalues if and only if $\theta$ in the equality (1) is also the eigenvalue $\lambda$ of $H$, using (5) the result follows.

The above conditions are satisfied by an infinite number of strongly regular graphs, and thus, there is an infinite number of cones with three distinct eigenvalues where two of them are main. The strongly regular graphs previously mentioned are examples satisfying these conditions.

Remark 2 Applying Theorem 1 (item 2) to the case of primitive strongly regular graphs $H$ with parameters $(n, k, a, c)$ and distinct eigenvalues $k>1>\lambda$, it follows that $K_{1} \nabla H$ has three distinct eigenvalues $\rho>1>\lambda$ if and only if $n=(2 k-c)(k-c)$ and $k=$ $2 c-a+1$. However, from Cheng et al. (2016, Lem. 4.9), we know that the cone over the Petersen graph is the unique cone over a strongly regular graph with three distinct eigenvalues $\rho>1>\lambda$. Therefore, without resorting to the classical feasibility conditions for strongly regular graphs [see (Brouwer and Haemers 2012, Th. 9.1.3)], we may conclude that there are no primitive strongly regular graphs distinct from the Petersen graph with parameters $((2 k-c)(k-c), k, a, c)$, where $k=2 c-a+1$.

Corollary 1 Let $H$ be a primitive strongly regular graph with parameters $(n, k, a, c)$ and distinct eigenvalues $k>\mu>\lambda$. Then:

1. $\lambda=-\mu$ if and only if $a=c$.

2. If $a=c$, then $K_{1} \nabla H$ has three distinct eigenvalues $\rho>\mu>\lambda$ if and only if $n+c=$ $k(1+\sqrt{k-c})$.

Proof 1. From (3) and (4), it is immediate that $\lambda=-\mu$ if and only if $a=c$.

2. Applying item 2 of Theorem $1, K_{1} \nabla H$ has three distinct eigenvalues $\rho>\mu>\lambda$ if and only if

$$
\begin{aligned}
\sqrt{k^{2}+4 n}-\sqrt{4(k-c)}=k & \Leftrightarrow \sqrt{k^{2}+4 n}=2 \sqrt{k-c}+k \\
& \Leftrightarrow k^{2}+4 n=4(k-c)+k^{2}+4 k \sqrt{k-c} \\
& \Leftrightarrow n=k-c+k \sqrt{k-c} \\
& \Leftrightarrow n=k(1+\sqrt{k-c})-c .
\end{aligned}
$$

According to Bridges and Mena (1979), a graph whose adjacency matrix is the incidence matrix of a multiplicative design is called a multiplicative graph. In Bridges and Mena (1979, Th. 4.1), it is stated that a connected graph $G$ is multiplicative if and only if it has three distinct 
eigenvalues $\rho>\mu>-\mu$. It should be noted that if $G$ has order $n$ and $\mu$ has multiplicity $p$, from $\rho+p \mu-(n-p-1) \mu=0$, it follows that $\rho=(n-2 p-1) \mu$. In addition, in the same paper, it is stated that when $G$ is a multiplicative cone over a graph $H$, either $H$ is a strongly regular graph with parameters $\left(\mu^{2}(\mu+2), \mu(\mu+1), \mu, \mu\right)$ or $G$ is a graph described therein. Now, using Corollary 1 , we furnish the following necessary and sufficient condition for obtaining multiplicative cones over connected $k$-regular multiplicative graphs.

Proposition 3 A cone over a connected $k$-regular multiplicative graph $H$ with three distinct eigenvalues $k>\mu>-\mu$ is multiplicative if and only if $H$ is strongly regular with parameters

$$
\left(\mu^{2}(\mu+2), \mu(\mu+1), \mu, \mu\right) .
$$

In such a case, the multiplicative graph $K_{1} \nabla H$ has distinct eigenvalues $\frac{k+\sqrt{k^{2}+4 n}}{2}>\mu>$ $-\mu$, where $k=\mu(\mu+1)$ and $n=\mu^{2}(\mu+2)$.

Proof Let $H$ be a connected $k$-regular multiplicative graph with three distinct eigenvalues $k>\mu>-\mu$. Then, $H$ is a primitive strongly regular graph, implying $\mu \neq 1$ and, taking into account Corollary 1 , it has parameters $(n, k, c, c)$. Still by Corollary 1 , the cone over $H$ has three distinct eigenvalues $\rho>\mu>-\mu$ if and only if $n+c=k(1+\sqrt{k-c})$, that is, applying (2) and (4), if and only if

$$
\begin{aligned}
\frac{k^{2}-\mu^{2}}{k-\mu^{2}}+k-\mu^{2}=k\left(1+\sqrt{k-k+\mu^{2}}\right) & \Leftrightarrow \frac{k^{2}-\mu^{2}}{k-\mu^{2}}=k(1+\mu)-k+\mu^{2} \\
& \Leftrightarrow(k-\mu)(k+\mu)=\mu(k+\mu)\left(k-\mu^{2}\right) \\
& \Leftrightarrow k-\mu=\mu\left(k-\mu^{2}\right) \\
& \Leftrightarrow k(1-\mu)=\mu(1-\mu)(1+\mu) \\
& \Leftrightarrow k=\mu(1+\mu) .
\end{aligned}
$$

Finally, from (2) and (6), it follows that $n=\mu(k+\mu)=\mu^{2}(\mu+2)$ and from (4) and (6), we obtain $c=k-\mu^{2}=\mu(1+\mu)-\mu^{2}=\mu$. The expression of $\rho$ is obtained taking into account (1).

The Shrikhande graph, which is a strongly regular graph with parameters $(16,6,2,2)$ and distinct eigenvalues $6>2>-2$, is an example of a multiplicative strongly regular graph with parameters $\left(\mu^{2}(\mu+2), \mu(\mu+1), \mu, \mu\right)$. Thus, its cone has the three distinct eigenvalues $8>2>-2$, where the main eigenvalues are 8 and -2 .

More general, the main eigenvalues of a multiplicative cone over a strongly regular graph with distinct eigenvalues $k>\mu>-\mu$ and parameters $\left(\mu^{2}(\mu+2), \mu(\mu+1), \mu, \mu\right)$ are $\rho=\frac{k+\sqrt{k^{2}+4 n}}{2}$ and $-\mu=\frac{k-\sqrt{k^{2}+4 n}}{2}$, according to Propositions 3 and 1 .

\subsection{Harmonic graphs}

A graph $H$ is said to be harmonic when $\mathbf{d}_{H}$ (the degrees vector of $H$ ) is an eigenvector associated with a (necessarily) integer eigenvalue, that is, if there is a positive integer $\ell$ such that $\mathbf{A d} \mathbf{d}_{H}=\ell \mathbf{d}_{H}$. It is immediate that every regular graph is harmonic. The harmonic graphs were introduced by Grünewald (2002) and Dress and Gutman (2003). In the paper by Nikiforov (2006), such a graph without isolated vertices is called pseudo-regular graph and it is defined as being a graph $H$, such that $\sum_{j \in N_{H}(i)} \frac{\mathrm{d}_{H}(j)}{\mathrm{d}_{H}(i)}$ is constant for every $i \in V(H)$.

The particular case of harmonic trees was studied by Grünewald (2002), where the author considers the trees $\mathscr{T}_{\ell}$, with $\ell \geq 2$, such that one of its vertices $v$ has degree $\ell^{2}-\ell+1$, 
while every neighbor of $v$ has degree $\ell$ and all the remaining vertices have degree 1 . He proved that these are the unique harmonic trees. These trees are among the ones with two main eigenvalues, which have been characterized by Hou and Zhou (2005) [see also Hou and Tian (2006)]. We may recall that a balanced double star is a tree with diameter equal to 3 in which the two non-pendent vertices have the same degree.

Proposition 4 (Hou and Zhou 2005) The stars, the balanced double stars, and the harmonic trees $\mathscr{T}_{\ell}$, for $\ell \geq 2$, are the unique trees with exactly two main eigenvalues.

Nikiforov (2006, Th. 8) proved that every main eigenvalue of an harmonic graph $H$ belongs to the set $\left\{-\lambda_{1}, 0, \lambda_{1}\right\}$. It is also stated in Nikiforov (2006, Th. 8) that if $H$ is a graph without a bipartite component, such that all main eigenvalues are in $\left\{-\lambda_{1}, 0, \lambda_{1}\right\}$, then it is harmonic. A similar result for connected graphs is obtained by Rowlinson (2007, Prop. 3.3), using a different approach. It is immediate to extend the spectral characterization of harmonic graphs without any restriction regarding their combinatorial structure, namely, considering arbitrary graphs (connected or not).

Proposition 5 A graph $H$ is harmonic if and only if every main eigenvalue of $H$ belongs to the set $\left\{0, \lambda_{1}\right\}$.

As immediate consequence from Proposition 1, it follows that there are no harmonic cones over regular graphs.

Now it is worth recalling the following reformulation of a result by Rowlinson (2007, Prop. 2.1).

Proposition 6 (Rowlinson 2007) If $G$ is a graph with $n$ vertices, $m$ edges, and exactly two main eigenvalues $\lambda_{1}>\lambda_{i}$, for some $i \in\{2, \ldots, n\}$, then

$$
\lambda_{i}=\frac{\sum_{j=1}^{n} d_{j}^{2}-2 m \lambda_{1}}{2 m-n \lambda_{1}} .
$$

The following proposition gives an alternative characterization of harmonic graphs.

Proposition 7 A graph $G$ with $n$ vertices and $m$ edges is harmonic if and only if $\lambda_{1}=\frac{\sum_{i=1}^{n} d_{i}^{2}}{2 m}$, and it has no more than two main eigenvalues.

Proof Suppose that $G$ is harmonic. By Proposition 5, all its main eigenvalues are in $\left\{0, \lambda_{1}\right\}$ and we have two cases: (i) $G$ is regular, with degree say $k$, and then, $\lambda_{1}=k$ is the unique main eigenvalue, or (ii) $G$ is non-regular, and then, it has two main eigenvalues. In case (i), $\lambda_{1}=k=\frac{n k^{2}}{n k}=\frac{\sum_{i \in V(G)} d_{i}^{2}}{2 m}$. And in the case (ii), by Propositions 6 and 5, it follows that $\lambda_{1}=\frac{\sum_{i \in V(G)} d_{i}^{2}}{2 m}$. Conversely, assume that $\lambda_{1}=\frac{\sum_{i \in V(G)} d_{i}^{2}}{2 m}$ and $G$ has no more than two main eigenvalues. If $G$ is regular, then the conclusion is immediate. If $G$ is not regular, by Proposition 6, the main eigenvalues of $G, \lambda_{i}$ and $\lambda_{1}$, are related by the equality (7). Replacing $\lambda_{1}$ in (7) by $\frac{\sum_{i \in V(G)} d_{i}^{2}}{2 m}$, it follows that the main eigenvalues of $G$ are in $\left\{0, \lambda_{1}\right\}$. Therefore, by Proposition 5, the result follows.

\section{The largest and the second largest eigenvalues of the complement of a graph}

The set of distinct main eigenvalues of the graph $G$ is herein called the main spectrum of $G$ and it is denoted MainSpec $(G)$. Therefore, the spectrum of $G$ is described as 
$\operatorname{Spec}(G)=\left\{\lambda_{1}^{\left[q_{1}\right]}, \ldots, \lambda_{p}^{\left[q_{p}\right]}\right\}$, where $\lambda_{j}^{\left[q_{j}\right]}$ means that the eigenvalue $\lambda_{j}$ has multiplicity $q_{j}$. Before proceeding, it is worth recalling the following fundamental result on the main spectrum of a graph.

Proposition 8 (Cvetković 1971) The sets MainSpec $(G)$ and MainSpec $(\bar{G})$ have the same number of elements. Furthermore, if $\lambda \in \operatorname{MainSpec}(G)$ and $\bar{\lambda} \in \operatorname{MainSpec}(\bar{G})$, then $\lambda+\bar{\lambda} \neq-1$.

Taking into account this result and the definition of main/non-main eigenvalue, it is immediate to obtain the basic results stated in the next proposition, partially proved by Hagos (2002) (see also Teranishi 2002).

Proposition 9 (Hagos 2002) Consider a graph $G$ and $\lambda \in \operatorname{Spec}(G)$. Then, the following assertions are equivalent:

(i) the eigenvalue $\lambda$ is either non-main or main with multiplicity greater than 1 ;

(ii) there is some eigenvector $\mathbf{v}$ of $G$ associated with $\lambda$, such that $\mathbf{j}^{\top} \mathbf{v}=0$;

(iii) the scalar $-1-\lambda$ belongs to $\operatorname{Spec}(\bar{G})$.

As direct consequence of this proposition, we may note that a necessary and sufficient condition for a simple eigenvalue $\lambda$ of a graph $G$ to be non-main is that $-1-\lambda$ to be an eigenvalue of $\bar{G}$ (see Hagos 2002).

Now, it is worth recalling the following consequence of Weyl's inequalities, the proof of which can be found in the book Cvetković et al. (1997):

$$
\lambda_{2}(\bar{G}) \leq-1-\lambda_{\min }(G) \leq \lambda_{1}(\bar{G}) .
$$

The relations (8) furnish a (negative) answer to the question raised in the paper Cardoso and Pinheiro (2009), about the existence of a graph $G$ for which the complement $\bar{G}$ has an eigenvalue less than its index and greater than $-1-\lambda_{\min }(G)$.

Proposition 10 If $G$ is a graph of order $n$ and $-1-\lambda_{\min } \neq \lambda_{1}(\bar{G})$, then $\bar{G}$ has no eigenvalues belonging to the open interval $\left(-1-\lambda_{\min }(G), \lambda_{1}(\bar{G})\right)$.

The inequalities (8) motivate us to consider graphs $G$ for which $-1-\lambda_{\min }(G)$ is an eigenvalue of $\bar{G}$. We have two cases: (a) $\lambda_{1}(\bar{G})=-1-\lambda_{\min }(G)$ and (b) $\lambda_{2}(\bar{G})=-1-$ $\lambda_{\min }(G)$.

In the case (a), we have that $-1-\lambda_{\min }(G)=\lambda_{1}(\bar{G})$ is a main eigenvalue of $\bar{G}$. Therefore, Proposition 8 guarantees that $\lambda_{\min }(G)$ is a non-main eigenvalue, since $-1=\lambda_{\min }(G)+$ $\left(-1-\lambda_{\min }(G)\right)$. In fact, regarding the equality (a), from (8) and Prop. 1.2 in Cardoso and Rowlinson (2010), we may conclude the following result.

Proposition 11 If $G$ is a graph, then $\lambda_{1}(\bar{G})=-1-\lambda_{\min }(G)$ if and only if the multiplicity of $\lambda_{1}(\bar{G})$ is greater than one.

As a consequence of Perron-Frobenius' Theorem, the condition $\lambda_{1}(\bar{G})=-1-\lambda_{\min }(G)$ in Proposition 11 implies that $\lambda_{\min }(G)$ is non-main and $\bar{G}$ is disconnected.

Example 1 The graph $G$ depicted in Fig. 1 is the one numbered 4 in Cvetković and Petrić (1984), from where we obtain that

$$
\operatorname{Spec}(G)=\{\mathbf{4 , 3 7 2 2 8}, 0,-1,-\mathbf{1 , 3 7 2 2 8},-2\},
$$

the numbers in bold being its main eigenvalues. Its complement $\bar{G}$ has $\operatorname{Spec}(\bar{G})=$ $\left\{\mathbf{1}^{[2]}, \mathbf{0}^{[2]},(-1)^{[2]}\right\}$, and we note that $-1-\lambda_{\min }(G)=-1-(-2)=1=\lambda_{1}(\bar{G})$. 
Fig. 1 A graph $G$ satisfying $\lambda_{1}(\bar{G})=-1-\lambda_{\min }(G)$ and its complement $\bar{G}$

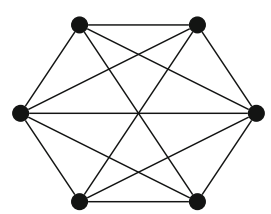

$G$

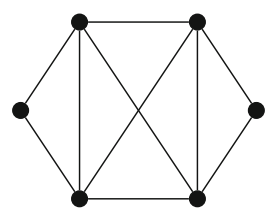

G

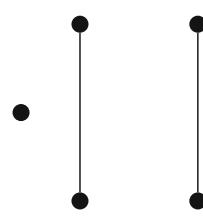

$\bar{G}$

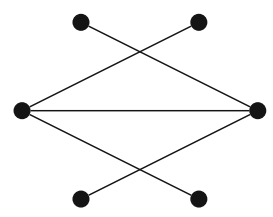

$\bar{G}$

On the other hand, regarding case (b), the following proposition is direct consequence of the above analysis.

Proposition 12 For any graph $G$, we have that $\lambda_{2}(\bar{G})=-1-\lambda_{\min }(G)<\lambda_{1}(\bar{G})$ if and only if $\lambda_{\min }(G)$ is main with multiplicity greater than one or it is non-main and $\lambda_{1}(\bar{G})$ is simple.

The inequalities in (8) and Propositions 11 and 12 allow us to conclude that if $\bar{G}$ is connected, then $-1-\lambda_{\min }(G) \in \operatorname{Spec}(\bar{G})$ if and only if $\lambda_{2}(\bar{G})=-1-\lambda_{\min }(G)$. This is the case of the graph $G$ depicted in Fig. 2, which is the graph number 23 of Cvetkovic and Petrić (1984). Indeed, $\operatorname{Spect}(G)=\left\{\mathbf{3 , 5 6 1 5 5}, 1, \mathbf{- 0 , 5 6 1 5 5},-1^{[2]},-2\right\}$ and $\operatorname{Spec}(\bar{G})=$ $\left\{\mathbf{2}, 1,0^{[2]},-\mathbf{1},-2\right\}$. We have $\lambda_{2}(\bar{G})=1=-1-\lambda_{\min }(G)$, with $\lambda_{2}(\bar{G})<\lambda_{1}(G)$.

From Proposition 9, for an arbitrary graph $G$ of order $n$, such that $\lambda_{\min }(G)$ is a simple eigenvalue, we have that $\lambda_{\min }(G)$ is non-main if and only if $-1-\lambda_{\min }(G)$ is an eigenvalue of $\bar{G}$. Particularly, within the class of bipartite graphs, we may conclude the following:

(a) For an arbitrary bipartite graph $G, \lambda_{1}(\bar{G})=-1-\lambda_{\min }(G)$ if and only if $\lambda_{\min }(G)$ is non-main and $\lambda_{1}(\bar{G})$ has multiplicity greater than one.

(b) If $G$ is connected and bipartite, then $\lambda_{2}(\bar{G})=-1-\lambda_{\min }(G)<\lambda_{1}(\bar{G})$ if and only if $\lambda_{\min }(G)$ is non-main and $\lambda_{1}(\bar{G})$ is simple.

The next result gives a combinatorial characterization of bipartite graphs $G$ of order $n$ for which $\lambda_{1}(\bar{G})=-1-\lambda_{\min }(G)$.

Theorem 2 Let $G$ be a bipartite graph. Then, $\lambda_{1}(\bar{G})=-1-\lambda_{\min }(G)$ if and only if $G$ is complete (bipartite) and balanced.

Proof Let us consider a bipartite graph $G$ with vertex set $V=V_{1} \dot{\cup} V_{2}$, where $\left|V_{1}\right|=r$ and $\left|V_{2}\right|=s$. If $\lambda_{1}(\bar{G})=-1-\lambda_{\min }(G)$, then (a) above implies that $\bar{G}$ is disconnected, and thus, $\bar{G}=K_{r} \dot{\cup} K_{s}$. Since $\lambda_{1}(\bar{G})$ is a multiple eigenvalue, then $r=s$. Conversely, if $G=K_{s, s}$, for some positive integer $s$, then $\bar{G}=\overline{K_{s, s}}$ is a disconnected graph with two components that are complete graphs with $s$ vertices. It follows that $\lambda_{\min }(G)=-s$ and $\lambda_{1}(\bar{G})=s-1$, and therefore, $\lambda_{1}(\bar{G})=-\lambda_{\min }(G)-1$. 


\section{Paths and double stars with non-main least eigenvalues}

Concerning the third question of Cardoso and Pinheiro (2009), we may note that among the connected graphs for which the least eigenvalue is non-main, we can count the harmonic graphs (see the Proposition 5), which class includes the regular graphs. In this section, the graphs with non-main least eigenvalue of two families of trees are characterized. We start by determining the paths with non-main least eigenvalue. For sake of completeness, we calculate the main spectrum of an arbitrary path.

It is worth recalling the following lemma which can be found in Cvetković et al. (1979), [the eigenvectors were described by Lee and Yeh (1993)].

Lemma 2 (Cvetković et al. 1979; Lee and Yeh 1993) For each integer $n$, the eigenvalues of $P_{n}$ are simple and given by $\lambda_{j}\left(P_{n}\right)=2 \cos \left(\frac{j \pi}{n+1}\right), 1 \leq j \leq n$. Each of these eigenvalues $\lambda_{j}$ has an associated eigenvector with entries $x_{i}=\sin \left(i \frac{j \pi}{n+1}\right)$, for $i \in\{1,2, \ldots, n\}$.

Theorem 3 For $n \geq 2$ and $1 \leq j \leq n, \lambda_{j}=2 \cos \left(\frac{j \pi}{n+1}\right)$ is a non-main eigenvalue of the path $P_{n}$ if and only if $j$ is even. In particular, the least eigenvalue of $P_{n}$ is non-main if and only if $n$ is even.

Proof Fix $j, 1 \leq j \leq n$. For the $\lambda_{j}$-eigenvector $\mathbf{v}^{(j)}=\left(x_{1}, \ldots, x_{n}\right)^{\top}$, we have $\lambda_{j} x_{i}=$ $\sum_{t \sim i} x_{t}$, whence $\lambda_{j} \sum_{i} x_{i}=\sum_{i} d_{i} x_{i}=2 \sum_{i} x_{i}-x_{1}-x_{n}$. From Lemma $2, \lambda_{j} \neq 2$, and then, $\sum_{i} x_{i}=0$ if and only if $x_{1}+x_{n}=0$. Since $x_{1}+x_{n}=2 \sin \left(\frac{j \pi}{2}\right) \cos \left(\frac{(n-1) j \pi}{2(n+1)}\right)$, we may verify that $\lambda_{j}$ is a non-main eigenvalue if and only if $j$ is even. Indeed, $\cos \left(\frac{(n-1) j \pi}{2(n+1)}\right)=0$ if and only if $\frac{(n-1) j \pi}{2(n+1)}=\frac{\pi}{2}+k \pi$ for $k \in \mathbb{N}$, and then, it follows that $1+2 k<j \leq n$. Also, it holds that $n=\frac{j+(1+2 k)}{j-(1+2 k)}$, which implies $n<\frac{2 n}{j-(1+2 k)}$, and then, $j<2 k+3$. Thus, $1+2 k<j<2 k+3$, which guarantees that $j$ is even.

Corollary 2 The path $P_{n}$ on $n$ vertices has $\left\lceil\frac{n}{2}\right\rceil$ main eigenvalues, where $\lceil x\rceil$ denotes the least integer not less than $x$.

A graph $G$ is said to be semi-regular bipartite if it is biregular and every edge joins a vertex of minimum degree to a vertex of maximum degree. The following result (see Rowlinson 2007) characterizes the semi-regular bipartite graphs in terms of their main eigenvalues.

Proposition 13 (Rowlinson 2007) A non-trivial connected graph $G$ is semi-regular bipartite if and only if its main eigenvalues are only $\lambda_{1}(G)$ and $-\lambda_{1}(G)$.

Combining Propositions 13 and 6, it follows that a connected semi-regular bipartite graph $G$ of order $n$ has $\lambda_{1}(G)=\sqrt{\frac{\sum_{i \in V(G)} d_{i}^{2}}{n}}$. This is a known result obtained in Hofmeister (1983), where it was stated that for a graph $H$ of order $n, \sum_{i \in V(H)} d_{i}^{2} \leq \lambda_{1}^{2}(H) n$ with equality if and only if $H$ is a semi-regular bipartite graph.

Since stars are connected semi-regular bipartite graphs, there exist no diameter- 2 tree with non-main least eigenvalue. Regarding diameter- 3 trees, it should be noted that these trees (the so-called double stars, see Fig. 3) are not semi-regular bipartite graphs, and then, combining Propositions 13 and 4, we may conclude that the least eigenvalue of a balanced double star 
Fig. 3 Double star $T(k, s)$

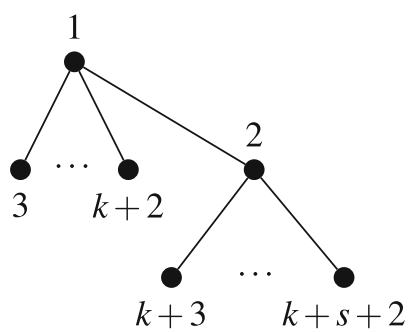

is non-main. On the other hand, we claim that there are no non-balanced double stars with least eigenvalue non-main.

To prove our assertion, we first introduce the notation $\mathbf{W}(\mathbf{B})$ to designate the matrix $\mathbf{W}(\mathbf{B})=\left[\mathbf{j} \mathbf{B} \mathbf{j} \mathbf{B}^{2} \mathbf{j} \cdots \mathbf{B}^{n-1} \mathbf{j}\right]$, where $\mathbf{B}$ is an arbitrary square matrix. When $\mathbf{B}=\mathbf{A}$, the adjacency matrix of $G, \mathbf{W}(\mathbf{A})=\left[w_{i j}\right]$ is such that $w_{i j}$ gives the number of walks in $G$ of length $j$ starting at vertex $i, 1 \leq i \leq n, 1 \leq j \leq n-1$, and then, it is called the walk matrix of $G$. A fundamental result due to Hagos (2002) [see also Powers and Suleiman (1982, Th. 4)] asserts that the rank of the walk matrix of $G$ is equal to the number of its main eigenvalues. To prove the next theorem, which includes our claim, we determine the main spectrum of a double star, combining the result obtained by Huang et al. (2015, Lem. 2.4) with the result due to Cvetković (1978, Th. 3). Note that, according to these results, the number of main eigenvalues of $G$ is equal to the rank of $\mathbf{W}(\mathbf{M})$, for $\mathbf{M}$ being the quotient (or divisor) matrix of any equitable partition of the vertex set of $G$, and the spectrum of $M$ includes the main eigenvalues of $G$.

Theorem 4 Let $T$ be a double star with $n$ vertices. If $T$ is balanced, then its two main eigenvalues are $\lambda_{1}$ and $\lambda_{n-1}$; otherwise, it has four main eigenvalues, namely $\lambda_{1}, \lambda_{2}, \lambda_{n-1}$, and $\lambda_{\min }$.

Proof Let $k \neq s$ be positive integers and $T=T(k, s)$ be a double star of order $n=k+s+2$ whose vertices are labeled as in Fig. 3.

Let us consider $V_{1}=\{1\}, V_{2}=\{2\}, V_{3}=\{3, \ldots, k+2\}$, and $V_{4}=\{k+3, \ldots, k+s+2\}$. Then, $V_{1} \dot{\cup} V_{2} \dot{\cup} V_{3} \dot{\cup} V_{4}$ is an equitable partition of $V(T)$ with associated quotient matrix:

$$
\mathbf{M}=\left[\begin{array}{llll}
0 & 1 & k & 0 \\
1 & 0 & 0 & s \\
1 & 0 & 0 & 0 \\
0 & 1 & 0 & 0
\end{array}\right]
$$

for which

$$
\mathbf{W}(\mathbf{M})=\left[\begin{array}{cccc}
1 & k+1 & k+s+1 & s+k^{2}+2 k+1 \\
1 & s+1 & k+s+1 & s^{2}+2 s+k+1 \\
1 & 1 & k+1 & k+s+1 \\
1 & 1 & s+1 & k+s+1
\end{array}\right] .
$$

It can be verified that $\operatorname{det} \mathbf{W}(\mathbf{M})=-k s(s-k)^{2}$, which is nonzero, since $s \neq k$. As $\mathbf{M}$ has characteristic polynomial $q(x)=x^{4}-(k+s+1) x^{2}+k s$ and, according to Del-Vecchio et al. (2009), the characteristic polynomial of $T$ is $p(x)=x^{s-1} x^{k-1}\left(x^{4}-x^{2}(k+s+1)+k s\right)$, we conclude that the four nonzero eigenvalues of the graph $T=T(k, s)$ are main when $k \neq s$. In particular, it follows that $\lambda_{\min }$ is a main eigenvalue (clearly, the others are $\lambda_{1}, \lambda_{2}$, and 
$\left.\lambda_{n-1}\right)$. On the other hand, when $k=s$, the tree $T=T(k, s)$ is a balanced double star that has exactly two main eigenvalues, according to Proposition 4 . In this case, the equitable partition of $V(T)$ has the only two cells $V_{1}=\{1,2\}$ and $V_{2}=\{3, \ldots, k+2, k+3, \ldots, 2 k+2\}$ and quotient matrix:

$$
\mathbf{M}=\left[\begin{array}{ll}
1 & k \\
1 & 0
\end{array}\right] .
$$

Applying a result due to Cvetković (1978, Th. 3), the two main eigenvalues of $T$ are the eigenvalues of $\mathbf{M}$, namely $\lambda_{1, n-1}=\frac{1 \pm \sqrt{1+4 k}}{2}$, as $\lambda_{\min }=-\lambda_{1}$. Therefore, our assertions are proved.

By combining Propositions 8 and 12 and Theorems 3 and 4, we may conclude immediately the next corollary.

Corollary 3 If $G$ is the path on $n$ vertices, then its complement has $\left\lceil\frac{n}{2}\right\rceil$ main eigenvalues and, for an even $n$, the second largest eigenvalue of $\bar{G}$ is equal to $-1-\lambda \min (G)$. In addition, the second largest eigenvalue of the complement of a balanced double star $T$ is equal to $-1-\lambda_{\min }(T)$.

Acknowledgements The authors would like to thank the anonymous referees for the constructive comments which help to improve the readability and quality of the paper. Domingos M. Cardoso would like to thank the hospitality of PEP/COPPE/UFRJ where this work began.

\section{References}

Allouch N (2017) The cost of segregation in (social) networks. Games Econ Behav 106:329-342

Bramoullé Y, Kranton R, D’Amours M (2014) Strategic interaction and networks. Am Econ Rev 104(3):898930

Bridges WG, Mena RA (1979) Multiplicative designs II. Uniform normal and related structures. J Combin Theory A 27:269-281

Brouwer AE, Haemers WH (2012) Spectra of graphs. Springer, New York

Cardoso DM, Pinheiro SJ (2009) Spectral upper bounds on the size of $k$-regular induced subgraphs. Electron Notes Discrete Math 32:3-10

Cardoso DM, Rowlinson P (2010) Spectral upper bounds for the order of a $k$-regular induced subgraph. Linear Algebra Appl 433(5):1031-1037

Cardoso DM, Sciriha I, Zerafa C (2010) Main eigenvalues and $(k, \tau)$-regular sets. Linear Algebra Appl 432:2399-2408

Cheng XM, Gavrilyuk A, Greaves G, Koolen J (2016) Biregular graphs with three eigenvalues. Eur J Comb 56:57-80

Cheng XM, Greaves G, Koolen J (2018) Graphs with three eigenvalues and second largest eigenvalue at most 1. J Combin Theory B 129:55-78

Chung H, Omidi GR (2009) Graphs with three distinct eigenvalues and largest eigenvalues less than 8. Linear Algebra Appl 430:2053-2062

Cvetković D (1970) The generating function for variations with restrictions and paths of the graph and selfcomplementary graphs. Univ Beograd Publ Elektrotehn Fak Ser Mat Fiz 320-328:27-34

Cvetković DM (1971) Graphs and their spectra. Univ Beograd Publ Elektrotehn Fak 354-356:1-50

Cvetković D (1978) The main part of the spectrum, divisors and switching of graphs. Publ Inst Math (Beograd) 23:31-38

Cvetković DM (1978) The main part of the spectrum, divisors and switching of graphs. Publications de L'Institut Mathématique 23(37):31-38

Cvetković D, Petrić M (1984) A table of connected graphs on six vertices. Discrete Math 50:37-49

Cvetković D, Doob M, Sachs H (1979) Spectra of graphs: theory and application. Academic Press, New York

Cvetković D, Rowlinson P, Simić S (1997) Eigenspaces of graphs, Encyclopedia of mathematics and its applications, vol 66. Cambridge University Press, Cambridge 
Cvetković D, Rowlinson P, Simić S (2010) An introduction to the theory of graph spectra. Cambridge University Press, Cambridge

Del-Vecchio RR, Gutman I, Trevisan V, Vinagre CTM (2009) On the spectra and energies of double-broom-like trees. Kragujevac J Sci 31:45-58

Dress A, Gutman I (2003) On the number of walks in a graph. Appl Math Lett 16:797-801

Grünewald S (2002) Harmonic trees. Appl Math Lett 15(8):1001-1004

Hagos EM (2002) Some results on graph spectra. Linear Algebra Appl 356:103-111

Hayat S, Koolen JH, Liu F, Qiaod Z (2016) A note on graphs with exactly two main eigenvalues. Linear Algebra Appl 511:318-327

Hayat S, Javaid M, Koolen JH (2017) Graphs with two main and two plain eigenvalues. Appl Anal Discrete Math 11:244-257

Hofmeister M (1983) Spectral radius and degree sequence. Math Nachr 139:211-222

Hou Y, Tian F (2006) Unicyclic graphs with exactly two main eigenvalues. Appl Math Lett 19:1143-1147

Hou Y, Zhou H (2005) Trees with exactly two main eigenvalues. Acta Hum Normal Univ 2(28):1-3 (in Chinese)

Huang X, Huang Q, Lu L (2015) Construction of graphs with exactly $k$ main eigenvalues. Linear Algebra Appl 486:204-218

Hu Z, Li S, Zhu C (2009) Bicyclic graphs with exactly two main eigenvalues. Ph.D. thesis

Lee S, Yeh Y (1993) On eigenvalue and eigenvectors of graphs. JMC 12:121-135

Lepović M (2001) Some results on graphs with exactly two main eigenvalues. Ph.D. thesis

Mena RA, Bridges WG (1981) Multiplicative cones: a family of three eigenvalue graphs. Aequationes Mathematicae 22:208-214

Muzychuk M, Klin M (1998) On graphs with three eigenvalues. Discrete Math. 189:191-207

Nikiforov V (2006) Walks and the spectral radius of graphs. Linear Algebra Appl 418:257-268

Powers DL, Sulaiman MM (1982) The walk partition and colorations of a graph. Linear Algebra Appl 48:145159

Rowlinson P (2007) The main eigenvalues of a graph: a survey. Appl Anal Discrete Math 1:455-471

Rowlinson P (2016) On graphs with just three distinct eigenvalues. Linear Algebra Appl 507:462-473

Rowlinson P (2017) More on graphs with just three distinct eigenvalues. Appl Anal Discrete Math 11:74-80

Shi L (2009) On graphs with given main eigenvalues. Appl Math Lett 22:1870-1874

Teranishi Y (2002) Main eigenvalues of a graph. Linear Multilinear Algebra 49:289-303

van Dam E (1998) Nonregular graphs with three eigenvalues. J Combin Theory B 73:101-118

Publisher's Note Springer Nature remains neutral with regard to jurisdictional claims in published maps and institutional affiliations. 